\title{
Extensive air showers with TeV-scale quantum gravity
}

\author{
Luis Anchordoqui, Haim Goldberg, Thomas McCauley, Thomas Paul, Stephen Reucroft, and John Swain \\ Department of Physics, Northeastern University, Boston, MA 02115, USA
}

\begin{abstract}
One of the possible consequences of the existence of extra degrees of freedom beyond the electroweak scale is the increase of neutrino-nucleon cross sections $\left(\sigma_{\nu N}\right)$ beyond Standard Model predictions. At ultra-high energies this may allow the existence of neutrino-initiated extensive air showers. In this paper, we examine the most relevant observables of such showers. Our analysis indicates that the future Pierre Auger Observatory could be potentially powerful in probing models with large compact dimensions.
\end{abstract}

Recently, it has become evident that a promising route to reconcile high energy particle physics and gravity is to modify the nature of gravitational interactions at distances shorter than a millimeter. Such a modification can be most simply achieved by introducing extra dimensions in the sub-millimeter range [1]. In this approach the fundamental scale of gravity $M_{*}$ can be lowered all the way to $\mathcal{O}(\mathrm{TeV})$, and the observed Planck scale turns out to be just an effective scale valid for energies below the mass of Kaluza-Klein (KK) excitations. Clearly, while the gravitational force has not been directly measured below the millimeter range, Standard Model (SM) interactions have been fairly well investigated at this scale; so if large extra dimensions really exist, one needs some mechanism to prevent SM particles from feeling those extra dimensions. Remarkably, there are several possibilities to confine SM fields (and even gravity) to a 4 dimensional subspace (referred to as a 3-brane) within the $(4+n)$ dimensional spacetime [2]. The provocative new features of this scenario have sparked a flurry of activity to assess its experimental validity. A brief resumé of current theoretical work devoted to higher dimensional models includes topics addressing fundamental issues of phenomenology [3], cosmology [4], astrophysics [5], and gravity [6]. Moreover, an intense effort to find signatures of extra-dimensions in collider data is currently underway |77.

Since 1966, a handful of extensive air showers have been observed corresponding to what seem to be single particles carrying over $10^{20} \mathrm{eV}$ [8]. This, in itself, is remarkable, as it is difficult or even impossible to explain how such energies can be attained by conventional acceleration mechanisms [9]. Deepening the mystery, it was pointed out by Greisen, Zatsepin and Kuz'min 10 (GZK) that extremely high energy ( $\left.\gtrsim 10^{20} \mathrm{eV}\right)$ cosmic rays, if nucleons and/or nuclei, would lose energy rapidly through interactions with the cosmic microwave background (CMB). This leads to the so-called GZK cutoff, which limits the propagation distance of these particles to roughly $50 \mathrm{Mpc}$. The difficulty in constructing nearby astrophysical sources that could accelerate particles to such high energies led to the belief that beyond roughly $10^{20}$ $\mathrm{eV}$, no cosmic rays would be detected. Adding to the puzzle, the arrival directions of these events are distributed widely over the sky, with no plausible optical counter- parts (such as sources in the Galactic plane or in the Local Supercluster). Furthermore, the "super-GZK" data are consistent with an isotropic distribution of sources in sharp contrast to the anisotropic distribution of light within $50 \mathrm{Mpc}$ from Earth 11]. In conclusion, the current picture is very unclear. Thus, it is reasonable to consider whether new physics could be at play.

Of particular interest here, the extraordinarily high center-of-mass (c.m.) energies achieved at the top of the atmosphere are well above those necessary to excite the hypothetical KK modes which would reflect a change in spacetime dimensionality 12]. Hence, a detailed analysis of extensive cosmic ray showers, taking into account this departure from previous fundamental particle theory, is worthwhile [13].

Interestingly enough, if gravity becomes strong at energies of a few $\mathrm{TeV}$, virtual graviton exchange can produce relatively large effects on the high energy scattering cross section, drastically changing the neutrino-nucleon interaction [14]. Neutrinos can propagate through the CMB essentially uninhibited, breaking the GZK barrier [15]. Unfortunately, within the SM scenario a neutrino incident vertically on the atmosphere would pass through it unihibited as well, never initiating an extensive air shower. It was already noted that within the extra dimensional framework, the neutrino nucleon cross section can approach typical hadronic values at c.m. energies $s \gtrsim 400 \mathrm{TeV}$, allowing earlier development of a vertical neutrino induced shower [16 18]. One may wonder whether the growth of the cross section carries with it observable deviations from SM predictions. Consistency with current experimental data requires [19,

$$
\sigma(E) \lesssim 3 \times 10^{-24} \frac{E}{10^{19} \mathrm{eV}} \mathrm{cm}^{2},
$$

and this bound certainly does not challenge the neutrinos acquiring a hadronic-scale cross section.

A complete theory of massive KK graviton modes is not yet available, making it impossible to know the exact cross section at asymptotic energies. Any air shower analysis would thus depend on reliable guesswork, supplemented with generally acceptable theoretical principles such as duality, unitarity, Regge behavior and parton structure. A simple Born approximation to the elastic $\nu$-parton cross section [17 (which underlies the total 
$\nu$-proton cross section) leads, without modification, to $\sigma_{\nu p}^{t o t} \sim s^{2}$. Unmodified, this behavior by itself eventually violates unitarity. This may be seen either by examining the partial waves of this amplitude, or by noting the high energy Regge behavior of an amplitude with exchange of the graviton spin-2 Regge pole: with intercept $\alpha(0)=2$, the elastic cross section

$$
\frac{d \sigma_{e l}}{d t} \sim \frac{\left|A_{R}(s, t)\right|^{2}}{s^{2}} \sim s^{2 \alpha(0)-2} \sim s^{2},
$$

whereas

$$
\sigma_{t o t} \sim \frac{\operatorname{Im}\left[A_{R}(0)\right]}{s} \sim s^{\alpha(0)-1} \sim s
$$

so that eventually $\sigma_{e l}>\sigma_{t o t}$. Eikonal unitarization schemes modify these behaviors: in the case of the tree amplitudes 14] the resulting (unitarized) cross section $\sigma_{\nu p}^{t o t} \sim s$, whereas for the single Regge pole exchange amplitude, $\sigma_{\nu p}^{t o t} \sim \ln ^{2}\left(s / s_{0}\right)$ [20]. However, the Regge picture of graviton exchange is not yet entirely established: both the (apparently) increasing dominance assumed by successive Regge cuts due to multiple Regge pole exchange 14 21], as well as the presence of the zero mass graviton can introduce considerable uncertainty in the eventual energy behavior of the cross section. Hereafter, we work within the unitarization framework of Ref. 14] and adopt as our cross section [22]

$$
\sigma_{\nu N} \approx \frac{4 \pi s}{M_{*}^{4}} \approx 10^{-28}\left(\frac{M_{*}}{\mathrm{TeV}}\right)^{-4}\left(\frac{E}{10^{19} \mathrm{eV}}\right) \mathrm{cm}^{2} .
$$

To simulate the consequences of this for $\nu$-induced air showers, we assume that the increase in the cross section is driven by the production of minijets [23], and we adopt the SIBYLL package to model the fragmentation region at ultra high energies 24]. In other words, the probability distribution for obtaining $N$ jet pairs (with $P_{T}^{\text {jet }}>P_{T}^{\text {min }}$, where $P_{T}^{\min }$ is a sharp threshold on the transverse momentum above which soft interactions are neglected) in a collision at energy $\sqrt{s}$ is computed regarding $\nu$-nucleon scattering as a diffractive shadow scattering associated with inelastic processes 25]. Particle production comes after the fragmentation of hypothetical colorless partonparton chains mimicking that of SIBYLL hadron-hadron scattering. The reader should keep in mind the crudeness of this approximation. However, the imposed cutoff on the soft processes ensures that the inelasticity in any neutrino-nucleon collision is not much larger than $y \sim 0.15$ 20], justifying the use of the SIBYLL package. As we discuss below, most of the expected qualitative features in the shower can be quite well reproduced. The algorithms of AIRES (version 2.1.1) [26] are slightly modified so as to track the particles in the atmosphere. In particular, Eq. (4) is translated into the neutrino mean free path

$$
\lambda_{\nu}=\frac{m_{\text {air }}}{\sigma_{\nu \text { air }}}
$$

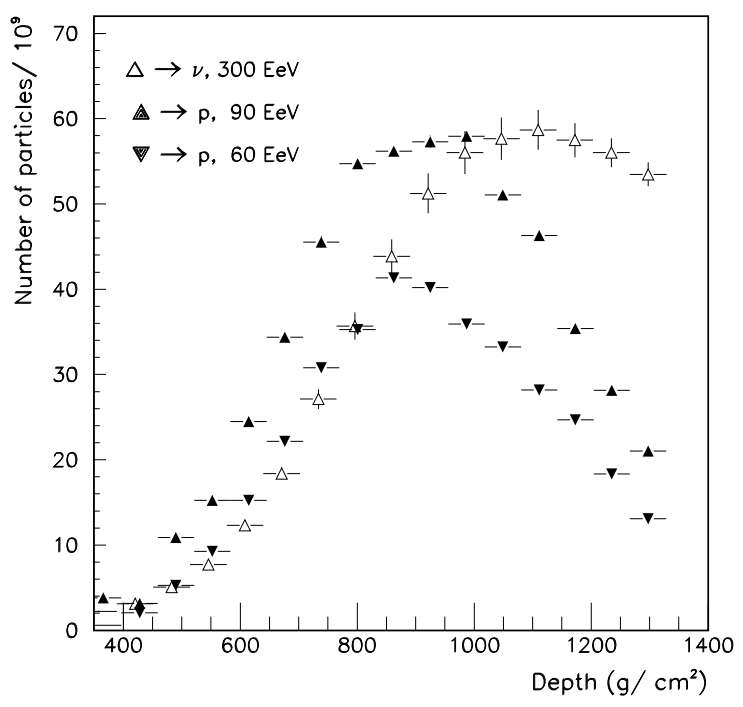

FIG. 1. Longitudinal development of neutrino and proton showers for different primary energies and primary zenith angle 43.9॰. The error bars indicate the standard fluctuations of the means.

via the standard 8 parameter function used in AIRES,

$$
\lambda_{\nu}=P_{1} \frac{1+P_{2} u+P_{3} u^{2}+P_{4} u^{3}}{1+P_{5} u+P_{6} u^{2}+P_{7} u^{3}+P_{8} u^{4}} \mathrm{~g} \mathrm{~cm}^{-2} .
$$

Here $m_{\text {air }}$ [g] is the mass of an average atom of air, and $u=\ln E[\mathrm{GeV}]$. The coefficients $P_{i}$ are listed in Table I for different values $M_{*}$.

Several sets of neutrinos were injected at $100 \mathrm{~km}$ above sea level. The sample was distributed in the energy range of $10^{20} \mathrm{eV}$ up to $10^{21} \mathrm{eV}$, and was uniformly spread in the interval of $0^{\circ}$ to $60^{\circ}$ zenith angle at the top of the atmosphere. All shower particles with energies above the following thresholds were tracked: $750 \mathrm{keV}$ for gammas, $900 \mathrm{keV}$ for electrons and positrons, $10 \mathrm{MeV}$ for muons, $60 \mathrm{MeV}$ for mesons and $120 \mathrm{MeV}$ for nucleons. The results of these simulations were processed with the help of the AIRES analysis package.

Figure 1 shows the total number of charged particles versus atmospheric depth averaged over 25 showers for the case of a $300 \mathrm{EeV}$ neutrino at $M_{*}=1 \mathrm{TeV}$. For comparison, proton-induced showers at 60 and $90 \mathrm{EeV}$ are shown on the same figure. As showers initiated by neutrinos typically start later than proton-induced showers, the longitudinal development tends to level off after reaching a maximum, in contrast to a standard air shower which decreases more rapidly after reaching a maximum. The number of charged particles produced in the cascade depends on the amount of energy deposited in the atmosphere by the primary. Neutrinos at the energy and mass scale shown in the figure typically suffer 2 interactions in 

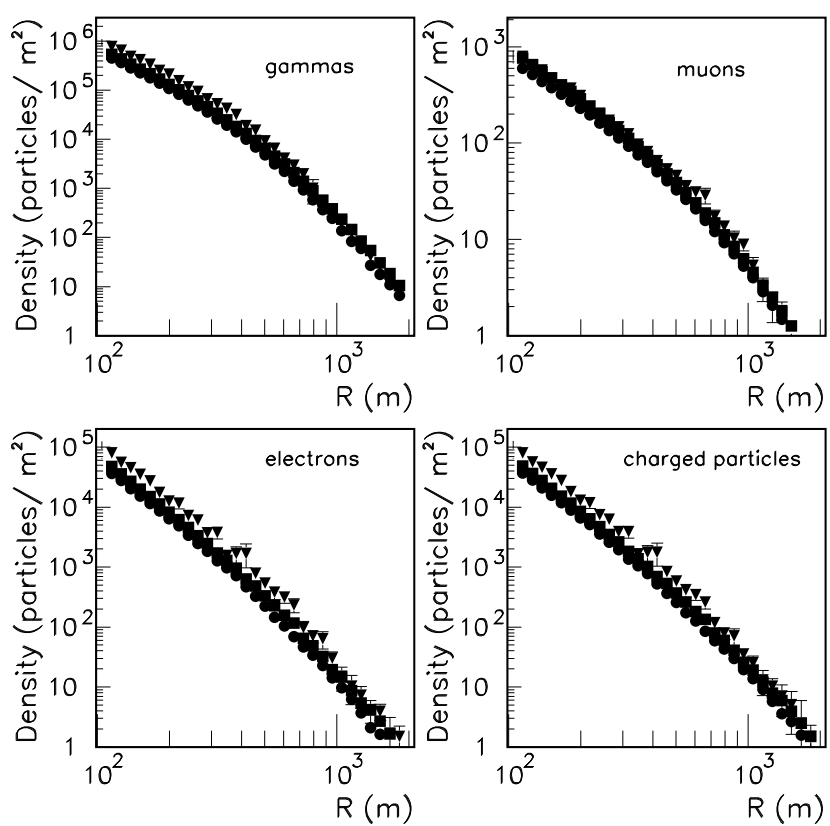

FIG. 2. Lateral distributions of vertical $300 \mathrm{EeV}$ neutrino-induced showers (triangles), $60 \mathrm{EeV}$ proton-induced showers (circles), and $60 \mathrm{EeV}$ iron-induced showers (squares). The error bars indicate the RMS fluctuations.

the atmosphere; any energy remaining after this is undetected. By comparing the neutrino-induced showers to the proton-induced showers shown in the figure, one can roughly estimate the inelasticity to be $0.1<y<0.15$. This is consistent with the estimates of reference 20 . *

Figure 2 shows the lateral distributions for vertical showers produced by $300 \mathrm{EeV}$ neutrinos, $60 \mathrm{EeV}$ protons, and iron nuclei of $60 \mathrm{EeV}$. At $50 \mathrm{~m}$ from the core, the ratio of the number of charged particles in the neutrino shower to that in the proton shower is $\approx 2$, whereas it is $\approx 1.5$ in $\nu /{ }^{56} \mathrm{Fe}$ showers. At about $1 \mathrm{~km}$ from the core these ratios reduce to $\approx 1.1$ and $\approx 0.7$, respectively. This is significant since experiments which rely on surface detectors to determine shower parameters typically use samples taken on the order of $1 \mathrm{~km}$ from the core, and thus would not be able to easily distinguish between these particle species.

Figure 3 shows the radial dependence of the mean arrival time of muons for showers initiated by $300 \mathrm{EeV}$ neutrinos and $60 \mathrm{EeV}$ protons. It can be readily seen from

*It is important to stress that the maximum number of charged particles produced in a proton-induced shower does not depend on the hadronic interaction model [27], making the present estimate on the inelasticity quite reliable.

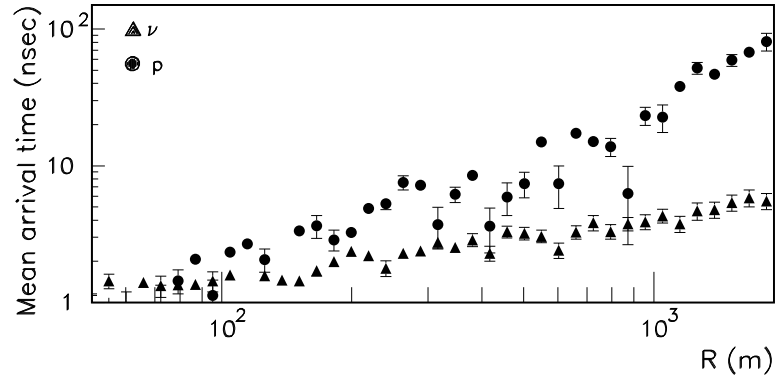

FIG. 3. Arrival times for charged particles in vertical $300 \mathrm{EeV}$ neutrino and $60 \mathrm{EeV}$ proton showers normalized at $50 \mathrm{~m}$ from the shower core. The error bars indicate the RMS fluctuations.

the comparison that the proton-induced showers exhibit larger fluctuations than the neutrino-induced showers. Besides, each profile presents a well defined slope that characterizes the shower front and comprises a signature of the primary species. In particular, a neutrino interacts in the atmosphere only once or twice, and consequently the muons reach the ground with a relatively short time delay.

The simulated neutrino showers discussed so far deposit far less energy in the atmosphere than the most energetic of the observed cosmic ray events. A natural question is then what the shower profile would look like for a neutrino whose energy and mean free path are such that it would deposit roughly the same energy as observed in the highest energy event 28].

At this stage, it is important to point out that within the SM framework neutrinos are produced at extremely high energies, typically by the weak decay of pions or other hadrons. Thus, one needs protons to be accelerated to energies a few orders of magnitude even higher. In scenarii involving precocious unification [29], there may be alternatives to decay chains for producing super-GZK neutrinos at the source.

Figure 4 shows the longitudinal development of a $900 \mathrm{EeV}$ neutrino-induced shower with a fundamental mass scale $M_{*}=1.3 \mathrm{TeV}$. We stress that such a scale is above the lower bound for $M_{*}$ derived from the expected flux of neutrinos and current non-observation of horizontal air showers [22]. The total energy deposited in the atmosphere (after 2 interactions) is of the same order as the Fly's Eye event, but the shower maximum occurs, as expected, significantly later.

In summary, it has been proposed [16 18] that the GZK cutoff can be skirted if the progenitors of the most energetic air showers are neutrinos. Under this hypothesis, the neutrino-nucleon cross section is increased by the presence of extra dimensions, allowing the neutrinos to interact in the atmosphere. Simulations indicate that neutrino-induced showers at energies of a few hundred EeV would exhibit signatures distinct from those of proton (or nucleus) induced showers that deposit a sim- 


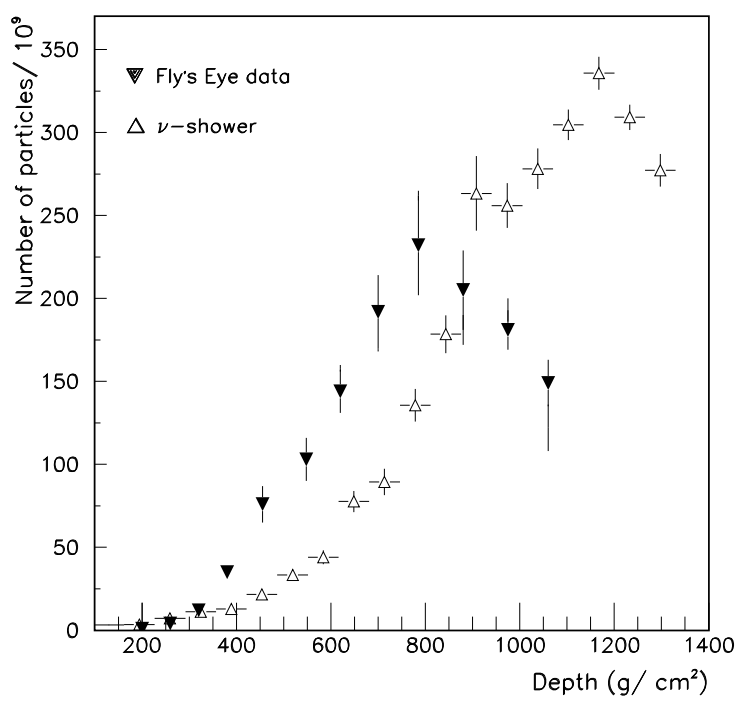

FIG. 4. The longitudinal development of a $900 \mathrm{EeV}$ neutrino-induced shower is shown together with the experimental data reported by Fly's Eye. The error bars in the simulated points indicate the standard fluctuations of the means.

ilar amount of energy in the atmosphere. Similarly, if there are neutrinos energetic enough to deposit as much energy in the atmosphere as is observed in the highest energy events, it appears they too may have unique signatures. In fact, any physics beyond the standard model that increases the neutrino-nucleon cross section should affect shower observables like longitudinal profile (measured with fluorescence detectors) and ground particle distributions (measured with surface detectors). This article contains some qualitative discussion of relevant observables of neutrino-induced showers. As far as we are aware, no showers have been observed which are consistent with these features. If candidates are eventually discovered, of course it will be necessary to carry out a much more detailed simulation than the one presented here. We note that future hybrid detectors such as the Pierre Auger Observatory [30] will be in an exceptional position to search for such phenomena.

Note added: After this paper was written, it was stressed that extremely high energy $(300 \mathrm{EeV})$ neutrinos with larger cross section ( $s^{2}$ rise) can create showers that would look like the highest energy event [31]. If this is the case, it should also be stressed that neutrinos of a few tens of EeV could induce vertical air showers with very distinctive profiles. In Fig. 5 we show the longitudinal development of showers initiated by neutrinos of $E=5 \times$
$10^{19} \mathrm{eV}$. f For comparison we also show showers induced by gamma rays and protons of $E=5 \times 10^{18} \mathrm{eV}$. It is easily seen that within this framework a $50 \mathrm{EeV}$ neutrino shower presents its own signature [32].

The question of whether the interaction cross section of neutrinos with matter could be greatly enhanced (via massive spin-2 exchange) at high energies, is yet undecided. Observation of deeply penetrating showers with $5 \times 10^{18} \mathrm{eV}$ deposited in the atmosphere would give an experimental and definite answer to this question. As an immediate spinoff, we have the converse fact, i.e., that if there were no possible candidate which could be associated with a neutrino shower, then it should be understood as a serious objection to the hypothesis of neutrinos as progenitors of the "super-GZK" events. We strongly recommend that the Fly's Eye data be reanalyzed searching for evidence of neutrino showers.

\section{ACKNOWLEDGMENTS}

We would like to thank Máximo Ave, Analía Cillis, Gabor Domokos, Michael Kachelrieß, Zurab Kakushadze, Susan Kovesi-Domokos, Jeremy Lloyd-Evans, Doug McKay, Michael Plumacher, John Ralston, Lisa Randall, Sergio Sciutto, Robert Shrock, and Ricardo Vázquez for useful discussions/correspondence. This work was partially supported by CONICET (Argentina) and the National Science Foundation.

[1] N. Arkani-Hamed, S. Dimopoulos and G. Dvali, Phys. Lett. B 429, 263 (1998); I. Antoniadis, N. Arkani-Hamed, S. Dimopoulos and G. Dvali, Phys. Lett. B 436, 257 (1998).

[2] G. Dvali and M. Shifman, Phys. Lett. B 396, 64 (1997), erratum ibid 407, 452 (1997); L. Randall and R. Sundrum, Phys. Rev. Lett. 83, 4690 (1999); B. Bajc, G. Gabadadze, Phys. Lett. B 474, 282 (2000); G. Dvali, G. Gabadadze, hep-th/0008054.

[3] See for instance, G. F. Giudice, R. Rattazzi and J. D. Wells, Nucl. Phys. B 544, 3 (1999); T. Han, J. D. Lykken and R. J. Zhang, Phys. Rev. D 59, 105006 (1999); J. L. Hewett, Phys. Rev. Lett. 824765 (1999); E. A. Mirabelli, M. Perelstein and M. E. Peskin, Phys. Rev. Lett. 82, 2236 (1999); T. G. Rizzo, hep-ph/9910255; S. Cullen, M. Perelstein and M. E. Peskin, Phys. Rev. D 62, 055012 (2000); L. Randall and R. Sundrum, Phys. Rev. Lett. 83,

\footnotetext{
${ }^{\dagger}$ To compute the simulation we adopt the cross section growth used in Ref. [31] to reproduce the Fly's Eye data.
} 


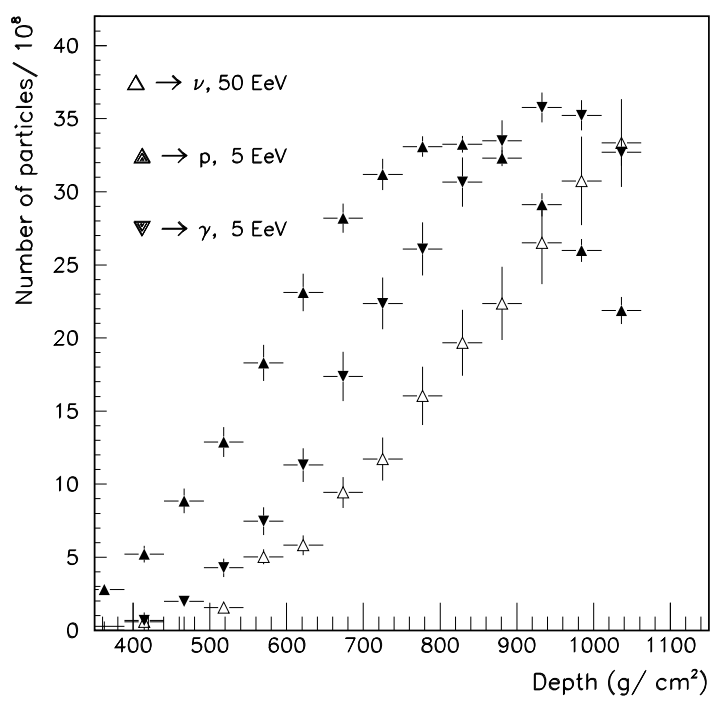

FIG. 5. Longitudinal development of $50 \mathrm{EeV}$ neutrino-induced showers, $5 \mathrm{EeV}$ proton-induced showers, and $5 \mathrm{EeV}$ gamma-ray showers. The error bars indicate the standard fluctuations of the means.

3370 (1999); J. Lykken and L. Randall, JHEP 0006, 014 (2000).

[4] See for instance, C. Csáki, M. Graesser, C. Kolda, J. Terning, Phys. Lett. B 462, 34 (1999); J. M. Cline, C. Grojean and G. Servant, Phys. Rev. Lett. 83, 4245 (1999); C. Csáki, M. Graesser, L. Randall and J. Terning, Phys. Rev. D 62, 045015 (2000); S. Nojiri and S. Odintsov, JHEP 0007, 049 (2000); L. A. Anchordoqui, C. Nuñez, K. Olsen, JHEP 0010, 050 (2000) hepth/0007064; S. W. Hawking, T. Hertog and H. S. Reall, hep-ph/0010232].

[5] See for instance, S. Cullen and M. Perelstein, Phys. Rev. Lett. 83, 268 (1999); N. Arkani-Hamed, S. Dimopoulos, G. Dvali and N. Kaloper, hep-ph/9911386]; V. Barger, T. Han, C. Kao, R. J. Zhang, Phys. Lett. B 461, 34 (1999); G. C. McLaughlin, Phys. Lett. B 470, 157 (1999); S. Cassisi, V. Castellani, S. Degl'Innocenti, G. Fiorentini, B. Ricci, Phys. Lett. B 481, 323 (2000).

[6] See for instance, W. D. Goldberger and M. B. Wise, Phys. Rev. Lett. 83, 4922 (1999) A. Chamblin, S. W. Hawking and H. S. Reall, Phys. Rev D 61, 065007 (2000); J. Garriga and T. Tanaka, Phys. Rev. Lett. 842778 (2000); S. Nojiri, S. D. Odintsov and S. Zerbini, Phys. Rev D 62, 064006 (2000); S. B. Giddings, E. Katz and L. Randall, JHEP 0003, 023 (2000); S. W. Hawking, T. Hertog and H. S. Reall, Phys. Rev. D 62, 043501 (2000); D. Langlois, R. Maartens and D. Wands, Phys. Lett. B 489, 259 (2000); C. D. Hoyle et al., hep-ph/0011014.

[7] M. Acciarri et al. (L3 Collaboration), Phys. Lett. B 470, 281 (1999) hep-ex/9910056; C. Adloff et al. (H1 Collaboration), hep-exp/0003002: B. Abbott et al. (DØ Collaboration), hep-ex/0008065.
[8] S. Yoshida and H. Dai, J. Phys G24 905 (1998); M. Nagano, A. A. Watson, Rev. Mod. Phys. 72, 659 (2000).

[9] For a comprehensive review on the origin of the highest energy cosmic rays the reader is referred to P. Bhattacharjee and G. Sigl, Phys. Rep. 327109 (2000).

[10] K. Greisen , Phys Rev. Lett. 16748 (1966); G.T. Zatsepin and V.A. Kuz'min, Pis'ma Zh. Eksp. Teor. Fiz. 4 114 (1966) [JETP Lett. 478 (1966)].

[11] The observed near-isotropy of the distribution can be explained, within a single-source hypothesis, postulating a galactic wind or a large extragalactic magnetic field. E. -J. Ahn, G. Medina-Tanco, P. L. Biermann and T. Stanev, astro-ph/9911123; G. Farrar and T. Piran, astro-ph/0010370. None of these models, however, could explain directional clustering as discussed by N. Hayashida et al. (AGASA Collaboration), Phys. Rev. Lett. 77, 1000 (1996); M. Takeda et al., Astrophys. J. 522, 225 (1999) astro-ph/9902239 and astroph/0008102]; Y. Uchihori et al., Astropart. Phys. 13, 151 (2000). For a recent analytic analysis, see H. Goldberg and T. J. Weiler, astro-ph/0009378.

[12] In considering the exchange of gravitons (KK modes), one should distinguish the following two scenarios: (i) In the canonical example of [1], KK gravitons are couple extremely weakly, and the observational effects arise because of the very large multiplicity of states due to their fine splittings. (ii) In the Anti-de Sitter bulk scenario (see Randall-Sundrum in Ref. 3|), each exited state coupling is $\mathcal{O}(E / \mathrm{TeV})$, and thus single KK modes could be detected via their decay products. Future cosmic ray data could play an important role in testing the latter. H. Davoudiasl, J. L. Hewett and T. G. Rizzo, hhep$\mathrm{ph} / 0010066$.

[13] The influence of the extra-dimensional scenario on monopole induced showers was reported elsewhere. L. A. Anchordoqui, T. P. McCauley, S. Reucroft and J. Swain, Phys. Rev. D 63, 027303 (2001) hep-ph/0009319.

[14] S. Nussinov and R. Shrock, Phys. Rev. D 59105002 (1999).

[15] A remarkable correlation between the arrival direction of cosmic rays above $10^{20} \mathrm{eV}$ and high redshift compact radio quasars seems to support the neutrino hypothesis. Such a correlation, however, diminishes when considering only the highest energy events $\left(E>8 \times 10^{19} \mathrm{eV}\right.$ at 1 standard deviation) that have no contamination from the expected proton pile-up around the photopion production threshold. G. R. Farrar and P. Biermann, Phys. Rev. Lett. 81, 3579 (1998); G. Sigl et al., astro-ph/0008363; A. Virmani et al., astro-ph/0010235.

[16] G. Domokos and S. Kovesi-Domokos, Phys. Rev. Lett. 82, 1366 (1999).

[17] P. Jain, D. W. McKay, S. Panda, and J. P. Ralston, Phys. Lett. B 484, 267 (2000).

[18] G. Domokos, S. Kovesi-Domokos and P. T. Mikulski hhep$\mathrm{ph} / 0006328$.

[19] H. Goldberg, T. J. Weiler, Phys. Rev. D 59, 113005 (1999).

[20] M. Kachelrieß and M. Plumacher, Phys. Rev. D 62, 103006 (2000) astro-ph/0005309.

[21] I. J. Muzinich and M. Soldate, Phys. Rev. D 37, 359, 
(1988).

[22] C. Taylor, A. Olinto and G. Sigl, Phys. Rev. D 63, 055001 (2001) hep-ph/0002257.

[23] T. K. Gaisser and T. Stanev, Phys. Lett. B 219, 375 (1989).

[24] R. S. Fletcher, T. K. Gaisser, P. Lipari and T. Stanev, Phys. Rev. D 50, 5710 (1994).

[25] L. Durand and H. Pi, Phys. Rev. Lett. 58, 303 (1987).

[26] S. J. Sciutto, in Proc. XXVI International Cosmic Ray Conference, (Edts. D. Kieda, M. Salamon, and B. Dingus, Salt Lake City, Utah, 1999) vol.1, p.411, astro$\mathrm{ph} / 9905185$.

27] L. A. Anchordoqui, M. T. Dova, L. N. Epele, S. J. Sciutto, Phys. Rev. D 59, 094003 (1999). See in particular Fig. 8.

[28] D. J. Bird et al., Astrophys. J. 441, 144 (1995).

[29] Z. Kakushadze, Nucl. Phys. B 548, 205 (1999); 552, 3 (1999); 551, 549 (1999). See also, 16 .

[30] For an overview of the Auger project see for instance, D. Zavrtanik, Nucl. Phys. B Proc. Suppl. 85, 324 (2000).

31] A. Jain, P. Jain, D. W. McKay, and J. P. Ralston, hep$\mathrm{ph} / 0011310$.

[32] It is worthwhile to remark that at these energies the CMB is completely opaque to the propagation of gamma rays. See for instance, R. J. Protheroe and P. A. Johnson, Astropart. Phys. 4, 253 (1996). 
TABLE I. Coefficients for mean free path parametrization

\begin{tabular}{cccccccc}
\hline \hline$M_{*}[\mathrm{TeV}]$ & $P_{1}$ & $P_{2}$ & $P_{3}$ & $P_{4}$ & $P_{5}$ & $P_{6}$ & $P_{7}$ \\
\hline 1 & -14657 & -2254.4 & -13.931 & 3.3530 & -1236.7 & -814.89 & -4.6945 \\
1.2 & 5654.4 & 1130000 & 1393 & -1417.3 & -1724000 & -124980 & 100.44 \\
1.3 & 6638.5 & 307640 & 355.94 & -366.14 & -1499700 & -19822 & 845.46 \\
\hline \hline
\end{tabular}

